\title{
Eficiência do mercado de capitais após a adoção da IFRS no Brasil: aplicando o teste de Mishkin
}

\author{
Capital market efficiency after IFRS adoption in Brazil: applying Mishkin test \\ Eficiencia del mercado de capitales después de adopción de IFRS en Brasil: la aplicación \\ de la prueba Mishkin
}

\section{Chrystian Tresman Zortea}

Mestre em Administração pela Fucape Business School

Endereço: Rua Sofia Jacob Miertchink, n॰217/103, Bairro Centro

CEP: 29645-000 - Santa Maria de Jetibá/ES - Brasil

E-mail: ctzfucape@hotmail.com

Telefone: (27) 99964-0997

\section{Fernando Caio Galdi}

Pós-Doutor em Contabilidade pela University of Arkansas/USA

Doutor em Controladoria e Contabilidade pela Universidade de São Paulo

Professor na Fucape Business School

Endereço: Av. Fernando Ferrari, n॰ 1358, Bairro Boa Vista

CEP: 29075-505 - Vitória/ES - Brasil

E-mail: fernando.galdi@fucape.br

Telefone: (27) 4009-4444

\section{Danilo Soares Monte-mor}

Doutor em Administração e Ciências Contábeis pela FUCAPE Business School

Professor na Fucape Business School

Endereço: Av. Fernando Ferrari, n॰ 1358, Bairro Boa Vista

CEP: 29075-505 - Vitória/ES - Brasil

E-mail: danilo@fucape.br

Telefone: (27) 4009-4444

\section{Aziz Xavier Beiruth}

Doutor em Controladoria e Contabilidade pela Universidade de São Paulo

Professor na Fucape Business School

Endereço: Av. Fernando Ferrari, n॰ 1358, Bairro Boa Vista

CEP: 29075-505 - Vitória/ES - Brasil

E-mail: aziz@fucape.br

Telefone: (27) 4009-4444

Artigo recebido em 31/05/2016. Revisado por pares em 14/03/2017. Reformulado em 13/04/2017. Recomendado para publicação em 26/05/2017 por Carlos Eduardo Facin Lavarda (Editor-Chefe). Publicado em 31/08/2017. 


\section{RESUMO}

Este estudo investiga se a eficiência do mercado de capitais brasileiro com relação à incorporação das informações contábeis aos preços se alterou após a adoção das normas internacionais de contabilidade (IFRS). Para tanto, aplica-se o teste de Mishkin (1983) visando testar a hipótese de eficiência de mercado. Os resultados indicam que para o primeiro período de análise (pré-IFRS) as variáveis explicativas, componentes do lucro em t tiveram um alto poder em inferir sobre os resultados do lucro futuro em $\mathrm{t}+1$. Entretanto, com os resultados obtidos no teste não foi possível afirmar sobre alguma mudança no cenário da eficiência do mercado acerca das informações contábeis no mercado de capitais após a adoção dos IFRS.

Palavras-Chave: Mercado de Capitais; Finanças; IFRS

\section{Abstract}

This study investigates the efficiency of the Brazilian capital market over the merger of accounting information to price change after the adoption of international financial reporting standards (IFRS). We apply the Mishkin test (1983) to test the market efficiency hypothesis. The results indicate that for the first period of analysis (pre-IFRS) the explanatory variables, income components in thave a high power to explain the results of future income in $t+1$. However, with the results obtained in the test it was not possible to say about any change in market efficiency scenario about the accounting information in the capital market after the IFRS adoption.

Abstract: Capital Market; Finance; IFRS

\section{Resumen}

Este estudio investiga la eficiencia del mercado de capitales brasileño sobre la fusión de la información contable a la variación de los precios después de la adopción de normas internacionales de información financiera (IFRS). Nosotros aplicamos el teste de Mishkin (1983) para poner a prueba la hipótesis de eficiencia del mercado. Los resultados indican que para el primer periodo de análisis (pre-IFRS) que las variables explicativas, componentes de la renta en $t$ tienen un alto poder inferir de los resultados de rentas futuras en $t+1$. Sin embargo, con los resultados obtenidos en la prueba de que no era posible decir acerca de cualquier cambio en el escenario de la eficiencia del mercado de la información contable en el mercado de capitales después de la adopción de las IFRS.

Palabras clave: Mercado de Capitales; Finanzas; IFRS

\section{Introdução}

Um dos pilares da teoria de finanças diz respeito à eficiência de mercado e à expectativa racional dos retornos, podendo, em tese, fornecer argumentos que possam explicar a alocação de recursos em um determinado ativo em função das informações disponíveis (BRUNI; FAMÁ, 1998).

Identificar o grau de eficiência de mercado torna-se importante, pois, dado que, a relação entre investidor e empresa é economicamente importante, quanto mais eficiente for o mercado de capitais, mais acessível será para o investidor que não possui ferramentas e tempo para analisar informações. Desta forma o mercado torna-se mais atraente, facilitando a captação de recursos para as empresas (MOURAD; PARASKEVOPOULOS, 2012). Nesse sentido, a modernização das regras e práticas contábeis, a criação do Novo Mercado e o CPC (Comitê de Pronunciamentos Contábeis) tornou o mercado de capitais brasileiro um ambiente mais propício para alocação de recursos (FORTI; PEIXOTO; SANTIAGO, 2009). 
De acordo com Niskaken (2016) o conceito de eficiência do mercado havia sido antecipado no início do século na dissertação de Jean Bachelier, apresentada à Universidade Sorbonne em 1900 para o seu PhD em matemática. Fama (1970) defende a hipótese de mercado eficiente (HME), que destaca que o retorno no mercado de ações é previsível e a existência de algum desvio do preço ou retorno anormal do ativo é rapidamente corrigida pelo mercado.

A arbitragem ocorrida no tempo de ajuste entre a informação publicada e a convergência dos preços dos ativos pode ser destacada como uma medida de mercado eficiente. Damodaran (2001) discute que a arbitragem pode ocorrer quando o ajuste dos preços em função da informação relevante é gradual. No entanto, algumas teorias contrárias a HME discutem que, no mercado de capitais a especulação e a assimetria de informação permitem retornos além dos esperados ou retornos anormais.

Shiller (2003) fundamenta suas ideias com uma opinião diametralmente oposta à teoria de mercados eficientes. $\mathrm{O}$ autor argumenta que os preços dos ativos possuem alguma relação com o comportamento do investidor e não uma mera causalidade entre a informação que é publicada e o ajuste dos preços. Shiller (2003) discute sobre a teoria das finanças comportamentais, que de forma ampla tem como pressuposto o objetivo de identificar e compreender como o comportamento dos investidores se afasta de um padrão, ou um comportamento probabilístico no qual o risco sistemático é ignorado.

A respeito da eficiência no mercado brasileiro, Barbosa e Camargos (2003) fazem uma revisão bibliográfica sobre o tema Eficiência de Mercado e encontram uma relação de artigos que apontam em sua maioria para o mercado eficiente na forma fraca.

De acordo com Santos (2011), o processo de globalização dos mercados de capitais, com ações sendo cotadas em diferentes mercados, incentiva a investigar a assimetria de informação nas demonstrações contábeis. Nesse sentido, as normas IFRS têm o objetivo de regulamentar a informação contábil, padronizar as normas já existentes e com isto mitigar a assimetria da informação entre os diferentes mercados (BATISTA et al., 2012).

Nessa ótica, cabe ressaltar a importância de responder a uma questão relevante: as novas normas contábeis com base nos padrões IFRS constituíram num aumento da eficiência no mercado de capitais brasileiro?

Buscou-se, por meio de um teste de racionalidade de mercado, responder a questão abordada acima. Inicialmente proposto por Mishkin (1983), o teste de racionalidade de mercado argumenta que a base para que o mercado seja eficiente fundamenta-se na premissa de que:

A eficiência do mercado implica que os preços dos títulos em um mercado de capitais devem refletir toda a informação disponível e, portanto, uma expectativa avaliada pelo mercado deve ser igual a verdadeira expectativa condicionada a todas as informações disponíveis. (MISHKIN, 1983)

A eficiência da informação no mercado de capitais é dada pela velocidade com que as informações disponibilizadas ao público se refletem nos preços das ações. Dessa forma, a presente pesquisa tem como proposta avaliar se as normas IFRS tiveram um impacto no mercado de capitais brasileiro e complementarmente avaliar a seguinte hipótese:

Ho Após a adoção das normas internacionais de contabilidade (IFRS) no Brasil, o mercado passou a ser mais eficiente com relação à incorporação das informações financeiras no preço das ações.

O desenvolvimento da área informacional na contabilidade deu-se por meio de questionamentos feitos à abordagem normativa, nos quais as conclusões sobre os processos contábeis eram dirigidas por um processo lógico-dedutivo. Dessa forma, uma nova abordagem, positivista, com análises baseadas mais no empirismo e em uma elaborada e deliberada interpretação individual, passa a ter credibilidade, devido ao seu processo estar baseado em 
dados e normas com direcionamento à essência do evento. Em detrimento desse aprimoramento, os estudos que relacionavam divulgação da informação contábil (lucro e patrimônio líquido) e a variação no mercado de capitais passam a ser mais intensos (LIMA, 2011).

\section{Referencial Teórico}

A relação entre as informações contábeis e a eficiência de mercado é amplamente discutida no arcabouço teórico. Galdi (2008) comenta que a demonstração contábil pode resultar em informações para tomada de decisão, no entanto, essa premissa está diretamente ligada ao nível de eficiência do mercado de capitais.

De acordo com Kothari (2001), a linha de pesquisa, mercado de capitais e demostrativos contábeis, teve como trabalho seminal a pesquisa de Ball e Brown na década de 1960. Lima (2011) comenta que esse estudo é conhecido por capital markets-based accounting research (CMBAR), pesquisa de contabilidade baseada em mercados de capitais.

Lewellen (2010) discute que o propósito da linha de pesquisa, mercado de capitais e demonstrativos contábeis, é entender como as demonstrações contábeis estão refletindo o valor da empresa e quão rápido essas informações direcionam os preços dos ativos em função dos demonstrativos contábeis. Essa abordagem, além de trazer entendimento na área da análise fundamentalista, também diz respeito aos trabalhos que focam a eficiência da informação.

Avaliar aspectos do mercado de capitais brasileiro, mais especificamente da eficiência da informação com o processo de harmonização das normas contábeis BR GAAP para as IFRS e as mudanças provocadas pela nova norma contábil, é, de alguma forma, uma revisão nos termos de eficiência de mercado e de expectativas nos retornos dos investimentos no mercado de capitais, dado que as informações disponíveis para o mercado sofreram modificações baseadas em um novo modelo contábil, as IFRS.

Pesquisas que relacionam lucro e preço das ações no mercado de capitais partem dos estudos de Ball e Brown (1968) e Beaver (1968), como destacam Macedo, Machado e Machado (2011). Dessa forma as pesquisas que relacionam indicadores contábeis e preço das ações vêm ganhando espaço, contribuindo com novas proxies, apresentando modelos de precificação e previsão do preço das ações. A adoção das normas internacionais ajudou na criação de campos de pesquisa, que buscam contrastar o cenário anterior e pós-IFRS.

Algumas pesquisas abordaram a importância ou impactos dos processos de harmonização e adequação às normas internacionais IFRS (BARBOSA; CAMARGOS, 2003; BATISTA et al. 2012; CALIXTO; SANTOS, 2010; LEMES; SILVA, 2007; LIMA, 2011; MACEDO; MACHADO; MACHADO, 2011). Os autores pesquisaram a relação do impacto no mercado em função da informação contábil, partindo da premissa de que as normas IFRS proporcionaram uma mudança no apreçamento dos ativos em função dos demonstrativos contábeis.

Dessa forma, as pesquisas sobre o mercado de capitais seguem uma tendência, buscando promover algum entendimento no que diz respeito as reformulações das informações contábeis e o impacto provocado no mercado de capitais.

No Brasil temáticas relativas às IFRS e mercado de capitais que pode ser destacadas são: evidenciação de informação contábil (BARBOSA; CAMARGOS, 2003); eficiência no mercado de capitais a adoção das normas IFRS (ANTUNES; GRECCO; FORMIGONI; NETO, 2012) ; componentes do lucro, persistência no lucro, anomalia dos accruals (BEUREN; COLAUTO, 2006; CUPERTINO; COSTA; MARTINEZ, 2011; FÁVERO; TAKAMATSU, 2012; GALDI, 2008); os impactos provocados pelas novas normas contábeis (ANTUNES; GRECCO; FORMIGONI; NETO, 2012; BATISTA et al., 2012; CALIXTO; SANTOS, 2010); 
e a relevância da informação nos processos de valuation e as IFRS (BEUREN; COLAUTO, 2006; GALDI, 2008).

No mercado Europeu, Naomi e Kevin (2007) encontram impactos positivos na harmonização contábil via normas IFRS, no entanto, discutem que os resultados encontrados não podem ser generalizados a outros mercados, pois cada país tem seu sistema jurídico e órgãos reguladores, a forma como o mercado local reage às informações, bem como a qualidade da informação reconhecida.

Algumas vantagens das IFRS citadas por Lima (2011) são que os países que convergem para as normas IFRS tendem a ter mais clareza nas informações contábeis, proporcionando, além da credibilidade, a integração com vários países e instituições que já aplicam esse método com uma linguagem comum. Ernst \& Young e Fipecafi (2008) comentam que a informação cabe representar o que de fato é verdade, sem efeitos ou manipulações, dando confiabilidade ao que foi exposto ao mercado. Dessa forma, o objetivo das informações é dar suporte para auxiliar na análise de risco inerente ao investimento e o retorno que ele produz.

Santana, Sarquis, Lourenço, Salotti e Murcia (2014) citam que a adoção das IFRS no Brasil aumentou a alocação de recursos e o potencial de diversificação dos portfólios de investimento. Os resultados deste estudo também mostraram que as condições de investimento no Brasil melhoraram, abrindo melhores oportunidades para investimentos estrangeiros no país, contribuindo para a globalização financeira e integração do mercado financeiro do país, o que destaca a importância macroeconômica do sistema de relatórios financeiros adotado.

Dado que a eficiência de informação no mercado de capitais torna-se um fator importante na tomada de decisão dos investidores, Cupertino, Costa Jr. e Martinez (2011) comentam que os investidores acompanham o resultado do mercado de capitais para apoiar suas expectativas com relação aos investimentos. De acordo com Damodaran (2001), a relação dos preços de mercado com a divulgação de informações ou eventos informativos tem sido uma das bases para comprovar a eficiência do mercado.

A eficiência da informação no mercado de capitais pode ser entendida como a velocidade com que as informações disponibilizadas ao público refletem nos preços das ações que, junto a outros fatores, indicam a eficiência informacional e traçam as expectativas dos investidores diante das informações (PEROBELLI; PEROBELLI; ARBEX, 2000).

Diante desse cenário de convergência das normas contábeis, cabe ressaltar o que de fato essas pontuações trouxeram de positivo para o Brasil. De acordo com Fávero e Takamatsu (2012, pág. 3), com o processo de harmonização das normas contábeis no Brasil baseado nas IFRS surgem oportunidades únicas em analisar o processo da informação contábil e como os agentes que demandam as informações para alocação de recursos usam a capacidade de interpretar os dados em função das novas normas contábeis "especialmente informações divulgadas concernentes ao lucro".

Biddle et al (2015) destaca que a adoção obrigatória das IFRS passou a ser significativamente associada com maior eficiência do investimento de capital medido pela sensibilidade do fluxo de investimento em dinheiro e pela tomada de risco por aumento de valor.

\section{Metodologia da Pesquisa}

\subsection{Coleta de Dados}

Com o objetivo de responder questão de pesquisa foram coletados dados de empresas, exceto as do setor financeiro e seguros, sendo o motivo dessa exclusão o fato de que estas "“[...] compõem setores regulamentados com legislação específica (portarias e circulares do Banco 
Central do Brasil, por exemplo) que impactam a contabilidade de maneira particular (RICHARDSON; TEOH; WYSOCKI, 1999, apud CUPERTINO; COSTA JR.; MARTINEZ, 2011, p. 6)".

Os dados que constituem a pesquisa foram extraídos na base de dados da Economática, e compreendem aos anos de 2005 a 2007 e aos anos de 2010 a 2013. Os anos de 2008 e 2009 foram excluídos da amostra porque este período representou um momento de transição na contabilidade brasileira, no qual o padrão adotado era um misto entre IFRS (adotado via alguns CPCs) e o BR-GAAP antigo ainda vigente.

\subsection{Modelo da Pesquisa}

Para verificar o aumento da eficiência no mercado de capitais brasileiro com a adoção das IFRS foi utilizado o Teste de Mishkin, segundo a Hipótese de Mercado Eficiente (HME). Dessa forma, buscou-se elucidar, com base no teste de Mishkin, os resultados obtidos com a adoção das normas IFRS no mercado de capitais brasileiro. Para a realização destes procedimentos são necessárias as seguintes informações contábeis: Retorno da firma no período $\mathrm{t}+1\left(R_{t+1}\right)$, Lucro no período $\mathrm{t}+1$ (lucros $\left._{t+1}\right)$, e os componentes do lucro no período $\mathrm{t}$ (accruals e fluxo de caixa).

As Variáveis utilizadas no teste estatístico foram o retorno anormal e o lucro (variáveis dependentes) e os componentes do lucro (variáveis explicativas), em períodos antes e pósadoção das normas IFRS.

\subsubsection{Cálculo do Retorno Anormal}

Para o cálculo dos retornos anormais, buscou-se utilizar-se da diferença do retorno normal (retorno de mercado) e o retorno esperado (CAPM) como mostra a equação abaixo:

$$
\operatorname{Ret}_{\text {anormal }}=\operatorname{Ret}_{\text {mercado }}-\operatorname{Ret}_{C A P M}
$$

A taxa de retorno de mercado é aquela que o investidor obtém como recompensa em relação a um investimento livre de risco. Essa taxa foi extraída do banco de dados do Economática.

O retorno esperado é calculado pelo método CAPM (Capital Asset Pricing Model) como verificado na equação abaixo:

Onde:

$$
E\left(R_{i}\right)=R_{f}+\beta \quad\left(R_{m}-R_{f}\right)
$$

$R_{f}$ : taxa livre de risco; usou-se, na presente pesquisa, a taxa SELIC.

$\beta$ : indicador de risco de cada ativo que representa o grau de variação de uma ação em relação ao índice IBOVESPA.

$R_{m}$ : taxa de retorno de cada ativo.

De acordo com Bruni e Famá (1998), o CAPM é uma das principais referências para o cálculo do retorno esperado, pois envolve o risco sistemático e retorno. Conjuntamente aplicado com a hipótese da eficiência de mercado, torna-se um dos principais conceitos da teoria de finanças.

\subsection{Teste de Mishkin}

De acordo com Leone, Kraft e Wasley (2007), o teste de Mishkin utilizado nas pesquisas 
contábeis testa a hipótese na qual a expectativa objetiva do mercado, baseado em informações passadas, é igual a expectativa subjetiva do mercado baseado em informações passadas. Sloan (1996) discute a eficiência de mercado na medida da importância dos retornos previsíveis das ações comparando com as previsões do modelo de expectativas de lucros naive, subjetivo são iguais.

A presente pesquisa contará com o teste de Mishkin, que aborda a questão eficiência de mercado e o apreçamento racional das ações no mercado de capitais. De acordo com Cupertino (2010),

Mishkin (1983) estabeleceu um teste de racionalidade e eficiência de mercado que consiste de um procedimento de estimação não linear por máxima verossimilhança. $O$ teste foi inicialmente concebido para testar a hipótese de expectativas racionais em macro econometria, fornecendo uma comparação estatística entre uma medida de precificação pelo mercado (coeficiente de avaliação) e outra de expectativas racionais (coeficiente de previsão), dada por uma variável relevante. (CUPERTINO, 2010).

O teste a ser usado nessa pesquisa avalia a condição de mercado eficiente com base nas medidas de lucro, com a seguinte hipótese (CUPERTINO, 2010; CUPERTINO; COSTA JR.; MARTINEZ, 2011):

Onde

$$
E_{m t}\left(\operatorname{lucros}_{t+1} \mid \emptyset_{t}\right)=E_{t}\left(\operatorname{lucros}_{t+1} \mid \emptyset_{t}\right)
$$

$\varnothing_{t}=$ conjunto de informações disponíveis no período $t$.

$E_{m t}\left(\right.$ lucros $\left._{t+1} \mid \emptyset_{t}\right)=$ expectativa subjetiva do mercado condicionado a $\emptyset_{\mathrm{t}}$.

$E_{t}\left(\right.$ lucros $\left._{t+1} \mid \emptyset_{t}\right)=$ expectativa objetiva do mercado condicionado a $\emptyset_{\mathrm{t}}$.

A eficiência de mercado atribui ao mercado retorno anormal igual a zero, conforme a equação abaixo:

Onde

$$
E_{t}\left(Y_{t+1}\right)=R_{t+1}-E_{m t}\left(\text { lucros }_{t+1} \vee \emptyset\right)=0
$$

$E_{t}\left(Y_{t+1}\right)=$ é o retorno anormal do período $\mathrm{t}+1$

$R_{t+1}=$ é o retorno do período $\mathrm{t}+1$

$E_{m t}\left(\right.$ lucros $\left._{t+1} \vee \emptyset\right)=$ é a expectativa subjetiva do mercado condicionado a $\varnothing_{t}$

De acordo com Cupertino (2010), o teste de Mishkin avalia se os investidores usam racionalmente as informações contidas nos lucros por meio de um procedimento de estimação não linear por máxima verossimilhança. Ainda de acordo com este autor, o teste busca estabelecer parâmetros entre as expectativas subjetivas e objetivas para a precificação das ações baseada nas informações sobre os lucros, contendo as informações passadas segundo as equações de regressão a seguir:

$$
\begin{gathered}
\operatorname{Lucros}_{t+1}=\alpha_{0}+\alpha_{1} \text { lucro }_{t}+v_{t+1} \\
R_{t+1}=\beta\left(\text { lucros }_{t+1}-\alpha_{0}-\alpha_{1} \text { lucros }_{t}\right)+e_{t+1}
\end{gathered}
$$

A primeira equação, de previsão, usa as informações do lucro no período t para prever $o$ lucro no período $t+1$. Na segunda equação, de precificação, o retorno é verificado em função das informações do lucro em $\mathrm{t}+1$ com a diferença das informações de lucro em $\mathrm{t}$.

De acordo com Sloan (1996); Cupertino, Costa Jr. e Martinez (2011), a estimação não linear das equações pode ser utilizada para entender como o mercado usa as informações do lucro em $\mathrm{t}$ para prever os lucros em $\mathrm{t}+1$. 
Ainda de acordo com Sloan (1996), os componentes do lucro, quando decompostos nas equações das regressões, têm o objetivo de inferir mais confiabilidade nas previsões acerca desses componentes, os accruals e fluxo de caixa, conforme as equações abaixo.

$$
\begin{gathered}
\operatorname{Lucros}_{t+1}=\gamma_{0}+\gamma_{1} F L C x_{t}+\gamma_{2} A C C r_{t}+v_{t+1} \\
R_{t+1}=\beta\left(\text { lucros }_{t+1}-\gamma_{0}-\gamma_{1} \text { FLC } x_{t}-\gamma_{2} A C C r_{t}\right)+\varepsilon_{t+1}
\end{gathered}
$$

Onde:

$F L C x_{t}=$ é o fluxo de caixa no período t;

$A C C r_{t}=$ são os Accruals no período t;

$v_{t+1}=$ erro;

$\varepsilon_{t+1}=$ erro.

Da mesma forma como exposto nas equações de previsão e precificação, os componentes do lucro foram decompostos segundo as medidas da contabilidade. O método não linear dos mínimos quadrados, aplicado nesse teste, impõe as restrições de acordo com a equação 10 , dado que a hipótese de que o mercado precifica racionalmente seus ativos está firmada nas igualdades dos coeficientes $F L C x_{t}$ e $A C C r_{t}$, tanto na equação de previsão, quanto na equação de equilíbrio de precificação.

Se o mercado precifica racionalmente os seus ativos, então:

$$
\gamma_{1}=\gamma_{1} \text { e } \gamma_{2}=\gamma_{2}
$$

Dessa forma, a eficiência de mercado ocorre quando o peso aplicado no $\gamma_{1}$, na equação de previsão, é o mesmo aplicado ao $\gamma_{1}$ Igualmente, o peso do $\gamma_{2}$, na equação de previsão, deve ser o mesmo na equação de equilíbrio de precificação, $\gamma_{2}$. Observando a equação 5 o retorno anormal não deve ser correlacionado com as informações passadas.

\section{ANÁLISE DOS RESULTADOS}

\subsection{Estatística Descritiva}

Nas Tabelas 1 e 2 são fornecidas as estatísticas descritivas das variáveis contábeis utilizadas nos testes, divididas em duas tabelas conforme o período analisado, antes e após as IFRS.

Tabela 1: Estatísticas descritivas anteriores a harmonização contábil

\begin{tabular}{lccc}
\hline \multicolumn{1}{c}{ Variável } & Média & Desvio Padrão & Mediana \\
\hline$\Delta$ Ativo Circulante & 0.0808 & 0.2359 & 0.0396 \\
$\Delta$ Caixa e Equiv. Caixa & 0.0205 & 0.0807 & 0.0038 \\
$\Delta$ Passivo Circulante & 0.1055 & 0.4699 & 0.0312 \\
$\Delta$ Finan. Dívida CP & 0.0207 & 0.1316 & 0.0043 \\
Depreciação & 0.0437 & 0.0496 & 0.0358 \\
$*$ Accruals & -0.0678 & 0.4417 & -0.0360 \\
$* *$ Fluxo de Caixa & 0.1768 & 0.4845 & 0.1463 \\
Lucro Operacional & 0.1186 & 0.2059 & 0.1139 \\
Retorno Anormal = CAPM-RM & 0.0696 & 0.0975 & 0.0714 \\
\hline
\end{tabular}

Nota: A amostra é constituída por empresas brasileiras cotadas na BOVESPA e disponíveis na base do Economática, referente aos anos de 2005 a 2007 (excluídas as empresas financeiras e de seguros) *Accruals definidos de acordo com o método de balanço e ** Fluxo de caixa calculado a partir da diferença entre os Accruals e Lucro Operacional. Os dados estão divididos pelo ativo total no início do período, excluídos os dados referentes ao retorno anormal. 
Verificam-se na Tabela 1 as variáveis que constituem os accruals conforme equação 3, o fluxo de caixa conforme equação 1, o lucro operacional e o retorno anormal. Quando comparados, a mediana dos accruals totais se aproxima dos encontrados em Sloan (1996) com mediana (3\%) e com Cupertino, Costa Jr. e Martinez (2011) com mediana de (3\%). Em relação aos dados sobre o Fluxo de caixa, no período observado, a mediana de $(11 \%)$ foi próxima a investigada por Sloan (1996) de (13\%) e mais distantes dos discriminados por Cupertino, Costa Jr. e Martinez (2011) de (8,6\%). Para o lucro operacional a mediana encontrada no período (11\%) foi a mesma observada por Sloan (1996), no entanto ao mercado brasileiro foi divergente a encontrada por Cupertino, Costa Jr. e Martinez (2011) com mediana de (4\%).

Verificam-se na Tabela 2 as variáveis que constituem os accruals conforme equação 3 , o fluxo de caixa conforme equação 1, o lucro operacional e o retorno anormal. De forma semelhante, os itens quando comparados ao período anterior a harmonização contábil, a mediana dos accruals totais se aproxima dos encontrados em Sloan (1996) com mediana (3\%) e com Cupertino, Costa Jr. e Martinez (2011) com mediana de (3\%). Em relação aos dados sobre o Fluxo de caixa, no período observado, a mediana de $(11 \%)$ foi próxima a investigada por Sloan (1996) de (13\%) e mais distantes ao discriminado por Cupertino, Costa Jr. e Martinez (2011) de (8,6\%). Para o lucro operacional a mediana encontrada no período (11\%) foi a mesma observada por Sloan (1996), no entanto ao mercado brasileiro foi divergente a encontrada por Cupertino, Costa Jr. e Martinez (2011) com mediana de (4\%).

Tabela 2: Estatísticas descritivas posteriores a harmonização contábil

\begin{tabular}{lccc}
\hline \multicolumn{1}{c}{ Variável } & Média & Desvio Padrão & Mediana \\
\hline$\Delta$ Ativo Circulante & 0.0446 & 0.1216 & 0.0357 \\
$\Delta$ Caixa e Equiv. Caixa & -0.0063 & 0.0856 & 0.0003 \\
$\Delta$ Passivo Circulante & 0.1130 & 1.3452 & 0.0291 \\
$\Delta$ Finan. Dívida CP & -0.0287 & 0.8322 & 0.0043 \\
Depreciação & 0.0346 & 0.0304 & 0.0324 \\
$*$ Accruals & -0.1379 & 1.1379 & -0.0183 \\
$* *$ Fluxo de Caixa & 0.2389 & 1.0814 & 0.1201 \\
Lucro Operacional & 0.1266 & 0.3865 & 0.0835 \\
Retorno Anormal = CAPM-RM & -0.0476 & 0.0973 & -0.0350 \\
\hline
\end{tabular}

Nota: A amostra é constituída por empresas brasileiras cotadas na BOVESPA e disponíveis na base do Economática, referente aos anos de 2010 a 2012 (excluídas as empresas financeiras e de seguros) *Accruals definidos de acordo com o método de balanço e ** Fluxo de caixa calculado a partir da diferença entre os Accruals e Lucro Operacional. Os dados estão divididos pelo ativo total no início do período, excluídos os dados referentes ao retorno.

\subsection{Análise dos Resultados}

A finalidade dessa pesquisa é testar os demonstrativos contábeis, como Cupertino, Costa Jr. e Martinez (2011); Leone, Kraft e Wasley (2007); Sloan (1996) Xie (2001), que aplicaram o teste de proposto por Mishkin (1983), para identificar anomalias no mercado de capitais ou comprovar as hipóteses subjacentes a eficiência de mercado. Na presente pesquisa o ponto de análise é a adoção das normas internacionais no mercado brasileiro.

De acordo com Batista, et al (2012) uma comparação entre os $\mathrm{R}^{2}$ dos resultados entre os dois períodos, anterior e posterior as normas IFRS, pode avaliar em qual período o poder explicativo e a significância do modelo são superiores, o que é demonstrado nas tabelas 03 e 04. Uma segunda comparação será feita entre os resultados dos testes de relação de probabilidade (Tabelas 05 a 08).

A Tabela 3 apresenta o resultado do ajuste geral do modelo antes da harmonização contábil. De acordo com Mishkin (1983) o teste segue a seguinte lógica: inicialmente as 
regressões são estimadas de forma irrestrita, sem que haja uma condição de igualdade entre os coeficientes, no segundo passo, a condição de igualdade dos coeficientes é imposta. A razão de máxima verossimilhança testa a igualdade dos coeficientes das duas equações, Lucro e Retorno Anormal, determinando a racionalidade de mercado. A hipótese nula que o mercado apreça racionalmente é constatada no teste $L R$ (likelihood ratio) com distribuição chi ${ }^{2}$.

Como mostra à estatística Chi2, tanto a equação de previsão quanto a equação de retorno foram significativas ao nível de 5\%. De acordo com as estimativas, $55,2 \%$ do lucro futuro $\mathrm{t}+1$ podem ser explicados pelos Accruals e Fluxo de caixa do período t. Já para retorno anormal do período $\mathrm{t}+1$ o ajuste geral do modelo foi aceito com o poder explicativo do modelo em 1,5\%, para afirmar que o retorno anormal é explicado pelas as variáveis independentes.

Tabela 3: Síntese das estimativas

\begin{tabular}{lccccc}
\hline Equação & DF & RMSE & R2 & Chi2 & P $>$ Chi2 \\
Previsão & 3 & 0.1385 & 0.5524 & 300.1 & 0.00000 \\
Retorno & 3 & 9.5247 & 0.0147 & 87.7 & 0.00000 \\
Observações & 153 & & & & \\
\hline
\end{tabular}

Nota. Fonte: Elaborado pelo autor

Os resultados no painel A da Tabela 4 indicam, na equação de previsão, a persistência média dos componentes do lucro, accruals e fluxo de caixa no lucro futuro. Os accruals (0.7709) e o fluxo de caixa representando (0.4135) também foi significante como mostra o (p valor, 0.0000). Pode-se notar que há uma persistência dos accruals em predizer o lucro futuro maior que o fluxo de caixa. No painel B da Tabela 4 , em relação à equação de precificação, fornece os resultados da persistência média dos componentes do lucro em relação à variável dependente retorno.

Tabela 4: Análise da regressão em painéis

\begin{tabular}{|c|c|c|c|c|}
\hline \multicolumn{5}{|c|}{ Dados referentes às observações no período de 2005 a 2007} \\
\hline \multicolumn{5}{|c|}{ Painel A - Equação de Previsão - } \\
\hline \multicolumn{5}{|c|}{ Lucros $_{t+1}=\alpha_{0}+\alpha_{1}$ Acccruals $_{t}+\alpha_{2}$ FluxodeCaixa $_{t}+v_{t+1}$} \\
\hline Variável & Coef. & Erro Padrão & Z-stat & $\mathrm{P}<|\mathrm{Z}|$ \\
\hline Constante $(\alpha 0)$ & 0.0972 & 0.0150 & 6.4630 & 0.0000 \\
\hline Accruals ${ }_{\mathrm{t}}$ & 0.7709 & 0.1037 & 7.4319 & 0.0000 \\
\hline Fluxo de Caixa ${ }_{\mathrm{t}}$ & 0.4135 & 0.0946 & 4.3723 & 0.0000 \\
\hline \multicolumn{5}{|c|}{$\begin{array}{l}\text { Painel B - Equação de precificação }-R_{t+1}=\beta\left(\text { lucros }_{t+1}-\alpha_{0}-\alpha_{1} \text { Acccruals }_{t}-\alpha_{2} \text { FluxodeCaixa }_{t}\right)+ \\
e_{t+1}\end{array}$} \\
\hline Variável & Coef & Erro Padrão. & Z-stat & $\mathrm{P}<|\mathrm{Z}|$ \\
\hline Lucro $t+1$ & 5.7651 & 5.5618 & 1.0366 & 0.2999 \\
\hline Constante $_{\alpha 0}$ & -1.0454 & 1.1168 & -0.9360 & 0.3493 \\
\hline Accruals ${ }_{t}$ & 0.3024 & 1.3177 & 0.2295 & 0.8185 \\
\hline Fluxo de Caixa $t$ & -0.2858 & 1.3148 & -0.2174 & 0.8279 \\
\hline
\end{tabular}

Nota. Fonte: Elaborado pelo autor

Os resultados, como vistos no painel B da Tabela 4, indicam que o Retorno anormal não é explicado pelo lucro e/ou informações passadas a respeito do lucro; diferentemente do ajuste geral do modelo que se mostrou significativo - chi2 $(87,7)$ - nenhuma das variáveis foi significativa em explicar o retorno anormal, o que de certa forma desperta interesse para futuras pesquisas, pois, diferentemente de outros achados, as variáveis têm, em certo grau, um poder elucidativo na equação de precificação, como demonstra Cupertino, Costa Jr. e Martinez (2011); Sloan (1996).

Diante dos resultados evidenciados nas Tabelas 3 e 4, o presente modelo torna-se limitado em responder se há eficiência de mercado ou não, ou seja, se o apreçamento por parte do mercado é racional ou não. 
$\mathrm{Na}$ Tabela 5, os resultados do teste de eficiência de mercado são fornecidos com base na equação (10) que é dada pela razão de máxima verossimilhança, distribuída assintoticamente $x^{2}(q)$ sendo (q) o número de restrições (SLOAN, 1996). O apreçamento racional é rejeitado se a estatística da razão de verossimilhança for suficientemente alta.

$$
2 \mathrm{n}_{\mathrm{x}} \operatorname{Ln}\left(\frac{S Q R r}{S Q R i}\right)
$$

Sendo n o número de observações, $\mathrm{L}_{\mathrm{n}}$ o logaritmo o operador do logaritmo natural, $\mathrm{SQR}^{\mathrm{r}}$ a soma dos quadrados dos resíduos restrita e $\mathrm{SQR}^{\mathrm{i}}$ a soma dos quadrados dos resíduos da regressão irrestrita. A Tabela 5 indica que os baixos coeficientes então dados pelo cálculo estatístico LR (likelihood ratio) com uma distribuição chi $^{2}$ (chi-quadrado) indicam que a diferença entre os coeficientes das duas regressões é não significativa, presumindo dessa forma o apreçamento racional do mercado.

Tabela 5: Análise dos dados da regressão

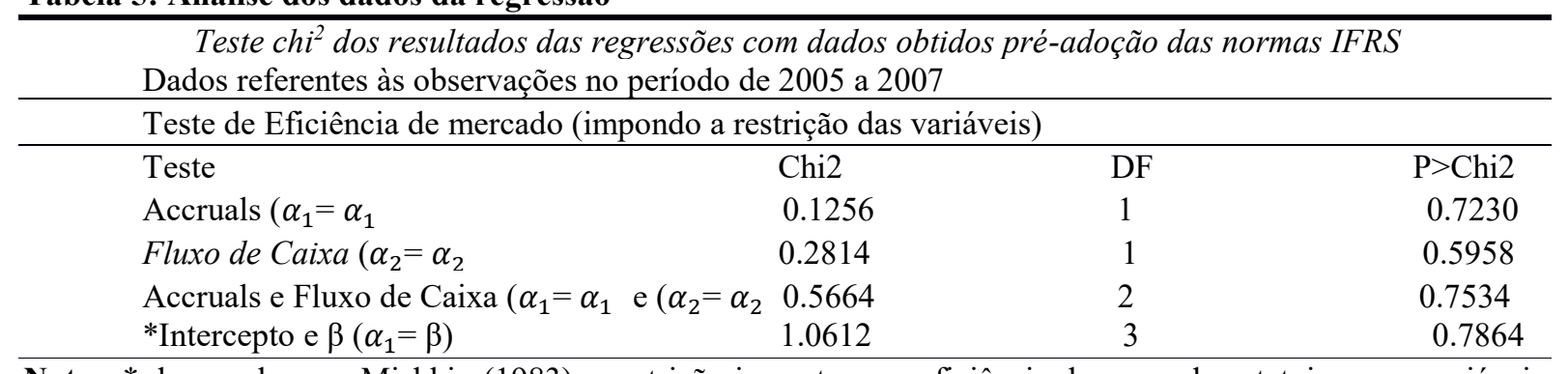

Nota $=*$ de acordo com Mishkin (1983) a restrição imposta para eficiência de mercado estatui que as variáveis Accruals e Fluxo de Caixa devem ser estatisticamente iguais.

A Tabela 6 remete ao período pós-harmonização contábil no mercado de capitais brasileiro tendo como referência as normas IFRS. Os resultados obtidos partem da mesma lógica utilizada nas tabelas anteriores, 3,4 e 5 .

A Tabela 6 apresenta o resultado do ajuste geral do modelo após a harmonização contábil. Como mostra à estatística Chi2, tanto a equação de previsão quanto a equação de retorno foram significativas ao nível de $5 \%$. Novamente, conforme observado no modelo antes a harmonização contábil, 53,3\% do lucro futuro podem ser explicados pelo Accruals e Fluxo de caixa do lucro presente. Também para o modelo que representa o retorno anormal, o ajuste geral do modelo foi significativo, incidindo poder de explicação de 5,64\%.

Tabela 6: Análise de regressão - Ajuste geral

\begin{tabular}{lccccc}
\hline Síntese das Estimativas & & & & & Chi2 \\
Equação & DF & RMSE & R2 & Chi2 \\
\hline Previsão & 3 & 0.0822 & 0.5336 & 350.0 & 0.00000 \\
Precificação & 3 & 0.0953 & 0.0564 & 47.2 & 0.00000 \\
Observações & 153 & & & & \\
\hline
\end{tabular}

Nota. Fonte: Elaborado pelo autor.

Os resultados no painel A da Tabela 7 indicam a persistência média dos componentes do lucro, accruals e fluxo de caixa no lucro futuro. Tanto os accruals $(0.4287)$ como o fluxo de caixa $(0.5098)$ foram significativos em relação ao lucro em $t+1$ como indica o ( $p$ valor, $0.0000)$.

Os resultados, como vistos no painel B da Tabela 7, indicam que o Retorno anormal não é correlacionado com o lucro e/ou informações passadas; nenhuma das variáveis foi significativa em explicar o retorno anormal. Novamente, como já observado nos resultados do modelo antes da harmonização contábil, o ajuste geral do modelo foi significativo - chi2 $(47,2)$. 
Da mesma forma o fato é curioso, pois, diferentemente de outros achados, as variáveis têm, em certo grau, um poder elucidativo na equação de precificação, como demonstra Cupertino, Costa Jr. e Martinez (2011) e Sloan (1996).

Tabela 7: Análise da regressão estatística

\begin{tabular}{|c|c|c|c|c|}
\hline \multicolumn{5}{|c|}{ Análise da regressão dos dados obtidos pós-adoção das normas IFRS } \\
\hline \multicolumn{5}{|c|}{ Dados referentes às observações no período de 2010 a 2012} \\
\hline \multicolumn{5}{|c|}{ Painel A - Equação de Previsão - } \\
\hline \multicolumn{5}{|c|}{ Lucros $_{t+1}=\alpha_{0}+\alpha_{1}$ Acccruals $_{t}+$ FluxodeCaixa $_{t}+v_{t+1}$} \\
\hline Variável & Coef. & Erro Padrão & Z-stat & $\mathrm{P}<|\mathrm{Z}|$ \\
\hline Constante & 0.0349 & 0.0093 & 3.7421 & 0.0002 \\
\hline Accruals & 0.4287 & 0.0530 & 8.0927 & 0.0000 \\
\hline Fluxo de Caixa & 0.5098 & 0.0557 & 9.1472 & 0.0000 \\
\hline \multicolumn{5}{|c|}{$\begin{array}{l}\text { Painel B- Equação de precificação - } \\
\qquad R_{t+1}=\beta\left(\text { lucros }_{t+1}-\alpha_{0}\right.\end{array}$} \\
\hline Variável & Coef & Erro Padrão. & Z-stat & $\mathrm{P}<|\mathrm{Z}|$ \\
\hline Lucro & -0.2087 & 0.0991 & -2.1073 & 0.0351 \\
\hline Constante & -0.1610 & 0.1064 & -1.5131 & 0.1303 \\
\hline Accruals & -0.0699 & 0.3776 & -0.1852 & 0.8531 \\
\hline Fluxo de Caixa & 0.0224 & 0.3865 & 0.0579 & 0.9538 \\
\hline
\end{tabular}

Nota. Fonte: Elaborado pelo autor.

A Tabela 8 indica que os baixos coeficientes então dados pelo cálculo estatístico LR (likelihood ratio) com uma distribuição chi $^{2}$ (chi-quadrado) indicam que a diferença entre os coeficientes das duas regressões, é não significativa, presumindo dessa forma o apreçamento racional do mercado. Nota-se que, os resultados evidenciados foram iguais aos resultados do modelo antes a harmonização contábil.

Tabela 8: Análise dos dados da regressão

Teste chi ${ }^{2}$ dos resultados das regressões com dados obtidos pré-adoção das normas IFRS

Dados referentes às observações no período de 2010 a 2012

Teste de Eficiência de mercado (impondo a restrição das variáveis)

\begin{tabular}{lccc} 
Teste & Chi2 & DF & P $>$ Chi2 \\
Accruals $\left(\alpha_{1}=\alpha_{1}\right.$ & 1.7102 & 1 & 0.1910 \\
Fluxo de Caixa $\left(\alpha_{2}=\alpha_{2}\right.$ & 1.5585 & 1 & 0.2119 \\
Accruals e Fluxo de Caixa $\left(\alpha_{1}=\alpha_{1}\right.$ e $\left(\alpha_{2}=\alpha_{2}\right.$ & 1.9967 & 2 & 0.3685 \\
*Intercepto e $\beta\left(\alpha_{1}=\beta\right)$ & 4.0101 & 3 & 0.2604 \\
\hline
\end{tabular}

Nota: Elaborado pelo autor

*De acordo com Mishkin (1983) a restrição imposta para eficiência de mercado estatui que as variáveis Accruals e Fluxo de Caixa devem ser estatisticamente iguais.

Em comparação com o período anterior as normas IFRS aplicadas ao mercado brasileiro, à persistência do lucro em prever o lucro futuro são menores. A Tabela 9 apresenta os resultados que comparam os coeficientes da equação de previsão para os dois períodos em relação a adoção das normas IFRS. Assume-se como hipótese nula a igualdade dos coeficientes. Ao nível de significância de 5\%, a persistência do lucro em prever o lucro futuro é menor após a harmonização contábil.

As principais mudanças chamam atenção à persistência dos accruals em relação ao fluxo de caixa. Uma diminuição desse primeiro componente pode indicar que as anomalias do mercado, como observadas em outras pesquisas (CUPERTINO; COSTA JR.; MARTINEZ, 2011; FÁVERO; TAKAMATSU, 2012; SLOAN, 1996; XIE, 2001) são menores quando esse componente representa menor expressividade em função da previsão do lucro futuro. 
Tabela 9: Comparação dos accruals e Fluxo de Caixa para os dois períodos

$\begin{array}{lcc}\text { Lucros }_{t+1}=\alpha_{0}+\alpha_{1} \text { Accrruals }_{t}+\text { FluxodeCaixa }_{t}+v_{t+1} & \\ \text { Variável } & \text { Coef. Anterior as normas IFRS } & \text { Coef. posterior as IFRS } \\ \text { Constante }(\alpha 0) & 0.0972 & 0.0349 \\ \text { Accruals }_{\mathrm{t}} & 0.7709 & 0.4287 \\ \text { Fluxo de Caixa } & 0.4135 & 0.5098\end{array}$

Nota: Elaborado pelo autor

\section{Considerações Finais}

Este estudo investigou a relação de eficiência no mercado de capitais brasileiro após a adoção das IFRS. Foram utilizados os componentes do lucro Fluxo de Caixa e Accruals como variáveis explicativas do lucro futuro e a relação entre os lucros futuros e a persistência no uso das informações dos componentes do lucro em função da variável retorno futuro.

Buscou-se aplicar o teste de Mishkin adaptado por Sloan (1996) para o cálculo estatístico com a proposta de extrair evidências diante das hipóteses levantadas. Com os resultados obtidos no teste não foi possível afirmar sobre alguma mudança no cenário das informações contábeis no mercado de capitais. Algumas observações a respeito das estatísticas podem ser analisadas quando comparadas a outros estudos. Comparando os resultados das estatísticas descritivas, pode-se notar que nos Accruals, a persistência desse componente do lucro em $\mathrm{t}$ como variável para a função dos lucros em $\mathrm{t}+1$ é menor para o período posterior a harmonização contábil.

Esse resultado, apesar de ser menos expressivo do que o teste proposto pode indicar que a Anomalia de mercado identificada por Cupertino, Costa Jr.e Martinez (2011) sofreu uma alteração depois da harmonização contábil dado que a influência dos accruals sobre os retornos dos ativos, como observada, foi menor.

Em relação ao arcabouço, eficiência de mercado de capitais para a análise dos retornos em função da adoção das normas internacionais IFRS, o tema ainda pode ser visto como fonte de muitas pesquisas, dado que a adoção das normas IFRS por todas as empresas de capital aberto só foi implantada a partir de 2010.

A limitação dessa pesquisa é dada principalmente ao tamanho da amostra. Comparando com outras pesquisas que usaram o teste de Mishkin para o mercado americano, Sloan (1996) observa mais de 40.000 empresas no período de trinta anos e no Brasil como verifica-se no trabalho de Cupertino, Costa Jr. e Martinez (2011) com um período de quase vinte anos.

Cabe ressaltar que o mercado brasileiro de capitais é carente de informações ou dados que permitam uma análise mais acurada de acordo com a metodologia aqui empregada. Como limitação dessa pesquisa pode-se expor o pequeno intervalo de tempo após a aplicação das normas IFRS para análise no mercado brasileiro. Outra limitação é o uso de apenas uma ferramenta nessa pesquisa, o que pode ser, para futuras pesquisas uma oportunidade de testar o mercado usando ferramentas distintas e comparar os resultados entre si, como por exemplo, Leone, Kraft and Wasley, (2007) que testaram o método (OLS) e o teste de Mishkin conjuntamente.

\section{Referências}

ANTUNES, M. T. P.; GRECCO, C. P.; FORMIGONI, H.; NETO, O. R. M. A adoção no Brasil das normas internacionais de contabilidade IFRS: o processo e seus impactos na qualidade da informação contábil. Revista de Economia e Relações Internacionais, São Paulo, v. 10, n. 20, p. 5-20, 2012, ISSN 1677-4973. 
BAESSO R.S.; COSCARELLI B.V.; AMARAL, F.V.A.; SILVA R.A.; AMARAL, H.F. Teste da Hipótese de Eficiência do Mercado no Brasil: uma Aplicação de Filtros Ótimos. In: Encontro Nacional de Pós-Graduação em Administração, 32., 2008, Rio de Janeiro. Anais... Rio de Janeiro: ENANPAD, 2008.

BALL, RAY; BROWN, PHILIP. An empirical evaluation of accounting income numbers. Journal of Accounting Research, p. 159-178, 1968.

BARBOSA, F. V.; CAMARGOS, M. A. Teoria e evidência da eficiência informacional do mercado de capitais brasileiro. Caderno de Pesquisas em Administração, São Paulo, v. 10, n. 1, 2003.

BATISTA, B. L. L.; et al. Análise do impacto do processo de convergência às normas internacionais de contabilidade no brasil: um estudo com base na relevância da informação contábil. In: Congresso USP de Controladoria e Contabilidade, 12., 2012, São Paulo. Anais... São Paulo, 2012.

BEAVER, William H. Alternative accounting measures as predictors of failure. The Accounting Review, v. 43, n. 1, p. 113-122, 1968.

BEUREN, I. M.; COLAUTO, R. D. Um Estudo sobre a Influência de Accruals na Correlação entre o Lucro Contábil e a Variação do Capital Circulante Líquido de Empresas. Revista de Administração Contemporânea, v. 10, p. 95-116, 2006. DOI http://dx.doi.org/10.1590/S1415-65552006000200006

Biddle, G. C.; Callahan, C. M.; Hong, H. A.; Knowles, R. L. Do Adoptions of International Financial Reporting Standards Enhance Capital Investment Efficiency? Available at SSRN 2353693, 2015.

BRUNI, A.L.; FAMÁ, R. Eficiência, previsibilidade dos preços e anomalias em mercados de capitais: teoria e evidências. Caderno de Pesquisa em Administração, São Paulo, v. 1, n. 7, p. 71-85, 1998.

CALIXTO, L.; SANTOS, E. S. Impactos do início da harmonização contábil internacional (Lei 11.638/07) nos resultados das empresas abertas. RAE-e, São Paulo, v. 9, n. 1, 2010 DOI http://dx.doi.org/10.1590/S1676-56482010000100006

DAMODARAN, Aswath. The Dark Side of Valuation: Valuing Old Tech, New Tech, and New Economy Companies. FT Press, 2001.

CUPERTINO, César Medeiros. Anomalia dos accruals no mercado brasileiro de capitais. 2010. Tese de Doutorado. Universidade Federal de Santa Catarina.

CUPERTINO, César Medeiros ; MARTINEZ, ANTÔNIO LOPO ; COSTA JR., NEWTON CARNEIRO AFFONSO DA. Accrual anomaly in the Brazilian capital market. BAR. Brazilian Administration Review , v. 9, p. 421-440, 2012.

ERNST \& YOUNG, F. Manual de normas internacionais de contabilidade: ifrs versus normas brasileiras. São Paulo: Atlas, 2008.

FAMA, F. E. Efficient capital markets: a review of theory and empirical work. Journal of Finance, New York, v. 25, n. 2, p. 383-417, 1970. DOI 10.1111/j.1540-6261.1970.tb00518.x.

FÁVERO, L.P.L; TAKAMATSU, R.T. Acrruals, persistência dos lucros e retorno das ações. In: Encontro Nacional de Pós-Graduação em Administração, 36., 2012, Rio de Janeiro. Anais... Rio de Janeiro: ENANPAD, 2012. 
FORTI, C.A.B.; PEIXOTO, F.M.; SANTIAGO, W.P. Hipótese de eficiência de mercado: um estudo exploratório no mercado de capitais brasileiro. Gestão \& Regionalidade, v. 25, n. 75, 2009. DOI 10.13037/gr.vol25n75.188.

GALDI, F. C. Estratégias de investimento em ações basedas na análise de demonstrações contábeis: é possível prever o sucesso? Tese (Doutorado) - Universidade de São Paulo, São Paulo, 2008.

KOTHARI, S. P. Capital markets research in accounting. Journal of Accounting and Economics, v. 31, p. 105-231, 2001. DOI http://doi.org/10.1016/S0165-4101(01)00030-1

LEMES, S.; SILVA, M. G. Experiência de Empresas Brasileiras na Adoção das IFRS. Contabilidade Vista \& Revista, v. 18, n. 3, p. 37-58, 2007.

LEONE, A.J.; KRAFT, A.; WASLEY, C. E. Regression-Based Tests of the Market Pricing of Accounting Numbers: The Mishkin Test and Ordinary Least Squares. Journal of Accounting Research, v. 45, n. 5, p. 1082-1114, 2007. DOI 10.1111/j.1475-679X.2007.00261.x.

LEWELLEN, J. Accounting anomalies and fundamental analysis: An alternative view. Journal of Accounting and Economics, v. 50, p. 455-466, 2010. DOI http://doi.org/10.1016/j.jacceco.2010.09.008.

LIMA, J. B. N. A relevância da informação contábil e o processo de convergência para as normas IFRS no Brasil. Tese (Doutorado em Ciências Contábeis) - Programa de Pósgraduação em Controladoria e Contabilidade, FEA/USP, São Paulo, 2011.

MACEDO, M.A.S.; MACHADO, M.A.V.; MACHADO, M.R. Análise da Relevância da Informação Contábil no Brasil num Contexto de Convergência às Normas Internacionais de Contabilidade. In: CONGRESSO NACIONAL DE ADMINISTRAÇÃO E CIÊNCIAS CONTÁBEIS - ADCONT, 2., 2011, Rio de Janeiro. Anais... Rio de Janeiro: ANCONT, 2011.

MISHKIN, F. S. A Rational Expectations Approach to Macroeconomics: Testing Policy Ineffectiveness and Efficient-Markets Models. University of Chicago Press, 2007.

MOURAD, A.N.; PARASKEVOPOULOS, A. IFRS: Introdução às Normas Internacionais de Contabilidade. 3. ed. Watson, 2012

NAOMI S. S.; KEVIN J.S. IFRS Adoption and Accounting Quality: A Review. European Accounting Review, v. 16, n. 4, p. 675-702, 2007. DOI http://dx.doi.org/10.1080/09638180701706732.

NISKANEN, S. Of thesis Accrual anomaly and accounting standards-Evidence from the adoption of IFRS. Journal of Financial Economics, v. 80, n. 3, p. 511-529, 2016.

PEROBELli F. F. C.; WALTER, L.N. Jr. Reações do Mercado Acionário a Variações Inesperadas nos Lucros das Empresas: um Estudo sobre a Eficiência Informacional no Mercado Brasileiro. In: In: Encontro Nacional de Pós-Graduação em Administração, 24., 2000, Florianópolis. Anais... Florianópolis: ENANPAD, 2000.

PEROBELLI, F. F. C.; PEROBELLI, F. S.; ARBEX, M. A. Expectativas Racionais e Eficiência Informacional: Análise do Mercado Acionário Brasileiro no Período 1997-1999. Revista de Administração Contemporânea, v. 4, n. 2, p. 07-27, 2000. DOI http://dx.doi.org/10.1590/S1415-65552000000200002

SANTANA, V. D. F. et al. Economic Effects of IFRS Adoption in Brazil: An Empirical Analysis of Stock Price Synchronicity. Available at SSRN 2383363, 2014. 
SANTOS, E. S. Full IFRS x Lei 11.638 ( $1^{\mathrm{a}}$ fase) x Lei 6.404: Impacto Esperado nos Resultados de 2010 a partir das Empresas que se anteciparam. In: CONGRESSO USP DE CONTROLADORIA E CONTABILIDADE, 11., 2011, São Paulo. Anais... São Paulo, 2011.

SANTOS, E.S.; CALIXTO, L. Impactos do início da harmonização contábil internacional (lei 11.638/07) nos resultados das empresas abertas. RAE-eletrônica, v. 9, n. 1, jan./jun. 2010.

SHILLER, R. J. From Efficient Markets Theory to Behavioral Finance. Journal of Economic Perspectives, v. 17, n. 1, p. 83-104, 2003. DOI 10.1257/089533003321164967.

SLOAN, R. G. Do Stock Prices Fully Reflect Information in Accruals and Cash Flows about Future Earnings? The Accounting Review, v. 71, n. 3, p. 289-315, 1996. DOI http://dx.doi.org/10.2469/dig.v27.n1.5

XIE, H. The Mispricing of Abnormal Accruals. The Accounting Review, v. 76, n. 3, p. 357$373,2001$. 